\title{
Study the Characteristics of Finite Impulse Response Filter Based on Modified Kaiser Window
}

\author{
Muzhir Shaban Al-Ani \\ Department of Computer Science, College of Science and Technology, University of Human Development, Sulaimani, \\ Kurdistan Region - Iraq
}

\begin{abstract}
A B S T R A C T
Finite impulse response (FIR) plays an important part between all other types of filters. There are many types windows used to design of FIR filters. Most important types are as follows: Hanning, hamming, rectangular, triangular, Blackman, Kaiser, etc. The characteristics of these filters depend on the number of generated coefficients in addition to the side lobes of the filter spectrum. The aim of this work is to study and evaluate Kaiser Window type depends on the variation of its factors applied for resizing the impulse response to reach a suitable size the filter. Kaiser Window is an important filter window that can be used to get many types of windows depending on their parameters. The proposed filter approach is designed and implemented through mixing of many filter factors. The filter characteristics are achieved using different values of filter size and attenuation. The implementation of the proposed Kaiser filter window provides an adequate and easy way to measure the window coefficients and maximum side lobe levels. The benefit of Kaiser Window that you can generate many types of window depending on the parameters change.
\end{abstract}

Index Terms: Filter Coefficients, Finite Impulse Response Filter, Kaiser Filter, Sidelobes

\section{INTRODUCTION}

There are two main categories of filters; infinite impulse response (IIR) and finite impulse response (FIR) [1]. These two categories of filters can be implemented for one-dimensional signals and two-dimensional (2D) signals, these filters can be implemented through software and hardware algorithms [2]. In addition, these can be implemented through time domain or frequency domain. Many of filter algorithms are implemented by hybridizing both software and hardware to achieve flexibility and

\section{Access this article online}

DOI: 10.21928/uhdjst.v1n2y2017.pp1-6

E-ISSN: $2521-4217$

P-ISSN: 2521-4209

Copyright (c) 2017 Al-Ani. This is an open access article distributed under the Creative Commons Attribution Non-Commercial No Derivatives License 4.0 (CC BY-NC-ND 4.0) real-time processing [3]. According to the characteristics of the impulse response, filters can be divided into four types; low-pass filter (LPF) that passes low frequencies, high-pass filter that passes high frequencies, band-pass filter that passes a band of frequencies, and band-stop filter that stop a band of frequencies [4]. The implementation of filter is realized through the convolution process between the input signal and the impulse response. To generate a FIR filter, the coefficients of time domain filter must be limited in number by multiplying by a window function of a finite width [5] The basic idea of applying windows through the design of filters is to truncate the sequence of the filter to be limited to certain values. Various types of windows are used for resizing the signal into limited values [6].

Many algorithms are implemented considering the factors of FIR filters to frequency domain specifications [7]. A narrow band LPF with good filter gain is introduced [8].

Corresponding author's e-mail: muzhir.al-ani@uhd.edu.iq

Received: 14-05-2017

Accepted: 19-06-2017

Published: 29-08-2017 
A hardware digital filter is implemented based on minimizing of computational expenses [9]. An adaptive analog filter is introduced based on echo cancelation [10]. An efficient pipelined filter is implemented using many structures [11]. A wideband efficient linear phase filter is implemented [12]. A high quality frequency domain applied in analog speech scrambler is reconstructed [13]. An efficient filter design based on FIR structure is implemented [14]. An optimized filter design technique is achieved [15]. A filter bank structure based on low complexity is implemented [16]. A programmable architecture applied programmable logic device (PLD) and FFT [17], then introduced PLD and digital signal processor [18]. Fast algorithm of filter design based on LUT is performed [19], then for 2D applications [20]. A real time digital filter processing based on hardware processor is realized [21].

\section{RELATED WORK}

Several researches have touched on this subject, the following are number of published works:

Mohindru et al. designed a new mathematical model to find the transfer function of LPF FIR based on Fourier transform and rotation angle. In contrast with the variation of the angle of rotation from 0 to $\pi / 2$, then it is possible to find the width of the transition band and the attenuation of the band. By raising the length of the filter, you can find the frequency response of the digital filter. The response characteristics of FIR filter can be organized through the operation, by controlling only one parameter and remaining other coefficients fixed [22].

Kumar et al. designed a novel approach for the structure of filters with variable band of finite linear impulse response band using different polynomials. In this work, the transfer function of a variable bandwidth filter, which is a linear combination of the linear phase filters with fixed coefficients and previous polynomials, is operated separately as control parameters to control the bandwidth of the filter. The proposed method also provides a better performance of tunable BPFs with larger filter captures compared to previously published results [23].

Pak et al. proposed a new approach to manage the horizon size of the nonlinear FIR filter. The proposed approach is to carry out an estimation of the state through a FIR filter bank. In this approach, the state estimate is obtained by weighting the average of several estimates of a bank of impulse responses using different horizon sizes. The filter size used for this approach is selected to maximize the likelihood function. The simulations of this approach gives good results compared with the conventional approach [24].

Leighton P. Barnes, George C. Verghese, studied the relationship between the Wiener filter and the coherence function, then defines the causal relationship between the wide-sense stationary (WSS) process. This causal consistency is interpreted in a modeling context and is used to show that a measure depends on the frequency of causality it can and cannot represent. In addition, the Wiener causal convergence of FIR filters with the Wiener causality of IIR filter is studied because the length of the filter passes the infinity. The main results show Lp convergence frequency responses under certain conditions of continuity support in the power spectra, and the upper asymptotic limits for the convergence error. Then, under the same conditions, the uniform convergence of AR approximations shows power spectra as the order model tends to infinity [25].

Huang et al. presented a filter design in the closed form based on the compensation of transfer characteristics. In this approach, a new filter design based on a convolution of window is presented and the relationship between window spectrum and filter performance is developed. Then, a schematic design of three step filter is designed these are designing an irregular filter, designing a compensation filter, and the sum of filter. This scheme can be simplified into a closed form characterized by two analytical formulas by merging the intermediate steps [26].

Pak et al. proposed an efficient nonlinear FIR. The proposed least square extended FIR filter is derived using a least squares criterion and one through the property. This approach is a special filter designed for the constant speed motion model and does not require noise information such as Gaussian noise covariance. If the noise information is very uncertain, this approach can provide a constant performance, whereas the non-linear estimators of the existing state, such as the extended Kalman filter and particle filter, degradation of performance often in the same condition. The simulations results indicated the robustness of this approach against the uncertainty of the noise model [27].

Boukharouba implemented a new technique for the FIR filters, where the desired frequency response is a smooth rectangular function. In this approach, go directly to smooth out the ideal desired response in the frequency domain not in time domain. The impulse response of the filter is a sampled 
version of the inverse Fourier transform of the frequency response. This approach achieved the best performance results of filter specifications compared with the results of other works [28].

\section{KAISER WINDOW DESIGN}

Kaiser Window have many parameters that affect the overall filter design. The ripple parameter $\alpha$ enabling the designer to trade-off the transition and ripple. It is defined in the interval $-\mathrm{M} \leq \mathrm{n} \leq \mathrm{M}$, and otherwise it is zero. The equations of the Kaiser Window and their parameters are shown below:

Measuring the ripple ( $\delta$ ) factor and then measure the attenuation factor $(A)$ as the following:

$A=-20 \log _{10} \delta$

Measure the value of $\alpha$ according to the value of attenuation (A) factor as the following:

$\propto=0.1102(A-8.7) A>50$

$\alpha=0.5842(A-21)^{0.4}+0.07886(A-21) 21 \leq A \leq 50$

$\alpha=0 A<21$

Find the window size $(M)$ according to the calculated attenuation value $(A)$ and the transition width (TW) as below:

$$
M \geq \frac{(A-7.95)}{28.72 * T W}
$$

Kaiser Window is calculated using the following equation:

$w(n)=\frac{I_{0}\left(\alpha \sqrt{1-(n / M)^{2}}\right.}{I_{0}(\alpha)} \quad-M \leq n \leq M$

And the zero order Bessel function $I_{0}(\alpha)$ is measured as below:

$I_{0}(x)=1+\sum_{n=1}^{\infty}\left[\left(\frac{x}{2}\right)^{n} \frac{1}{n !}\right]$

\section{PROPOSED FIR FILTER ALGORITHM}

There are many parameters affected the filter design, these parameters are ripple factor, attenuation factor, TW, number of coefficients, and window type in addition to the required accuracy.

The length of the filter concentrated on the number of values of impulse response samples in the FIR filter, then the impulse response is varied from $n=0$ to $n=M-1$, where $M$ is the filter length.

The proposed filter approach is demonstrated in Fig. 1 and is shown below:

Step 1: Ripple ( $\delta$ ) measurement, considering pass band ripple and stop band ripple.

Step 2: Attenuation measurement depending on the ripple value.

Step 3: TW measurement depending on the number of coefficients.

Step 4: Filter length measurement based on the TW.

Step 5: Bessel function $\mathrm{I}_{0}(\mathrm{x})$ measurement depending on the calculated parameters.

Step 6: Kaiser Window elements measurement.

Step 7: Filter coefficients measurement depending on the given cutoff frequency.

Step 8: Filter coefficients truncation based on the required Kaiser Window.

Step 9: Realize the filter according to the measured parameters.

\section{RESULTS AND DISCUSSIONS}

To realize and study the FIR filter characteristics, it is better to generate different types of windows. These windows are; Barthann, Bartlett, Blackman, Blackman-Harris, Bohman, Chebyshev, Flattop, Gaussian, Hamming, Hanning, Nuttall, Parzen, Rectangular, Taylor, Triangular, Tukey, and Kaiser. To study the shape characteristics of these windows, these are generated using different length number of window. Fig. 2 shows these windows in time domain and frequency domain generated using 20 values. Fig. 3 shows these windows in time domain and frequency domain generated using 50 values. These two figures illustrated that the time domain shape varying from rectangular shape to different type of Sinc function shape or hat shape. In addition, these two

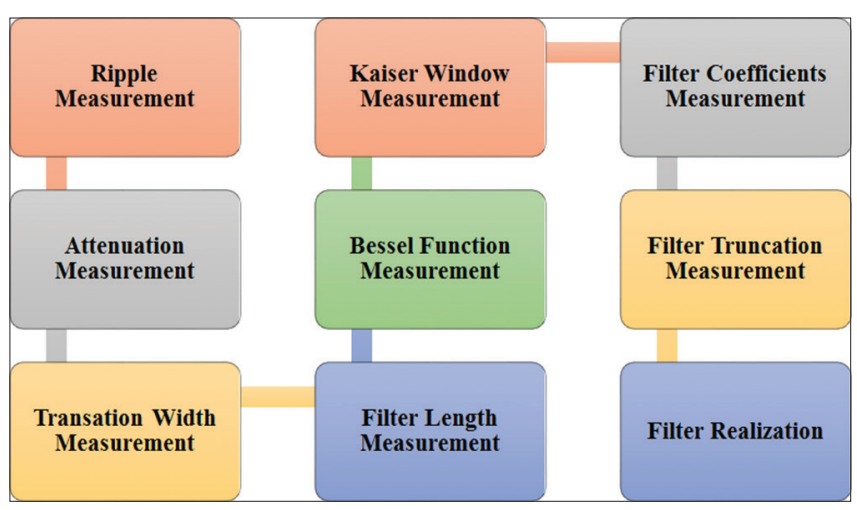

Fig. 1. Proposed finite impulse response filter algorithm 


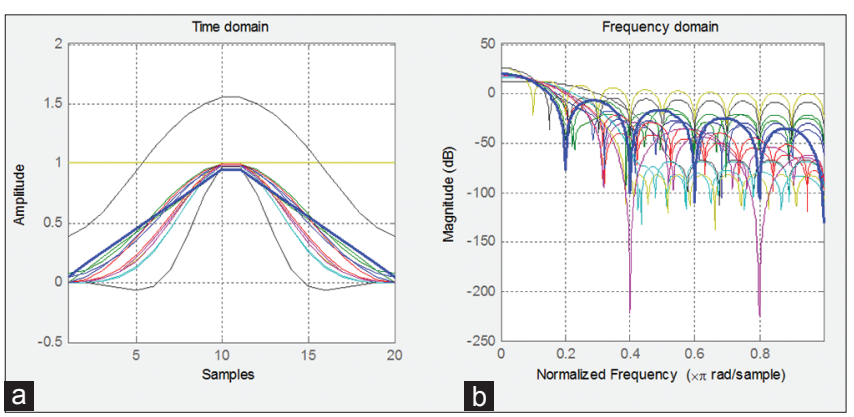

Fig. 2. $(a$ and b) Time domain and frequency domain representation of 20 values

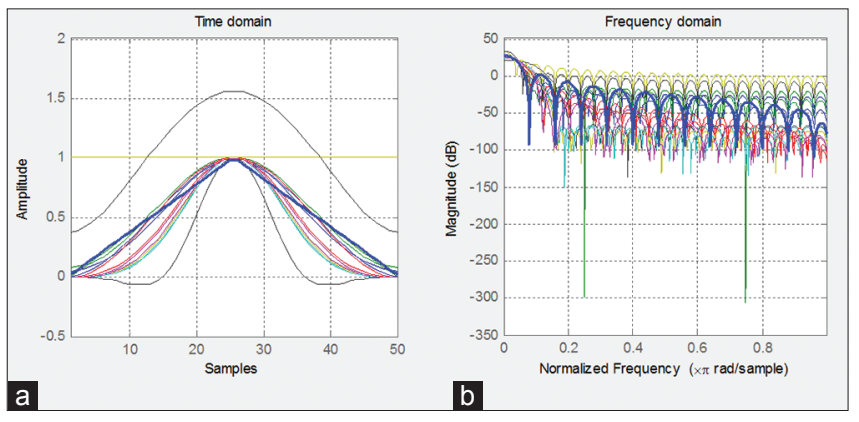

Fig. 3. ( $a$ and b) Time domain and frequency domain representation of 50 values

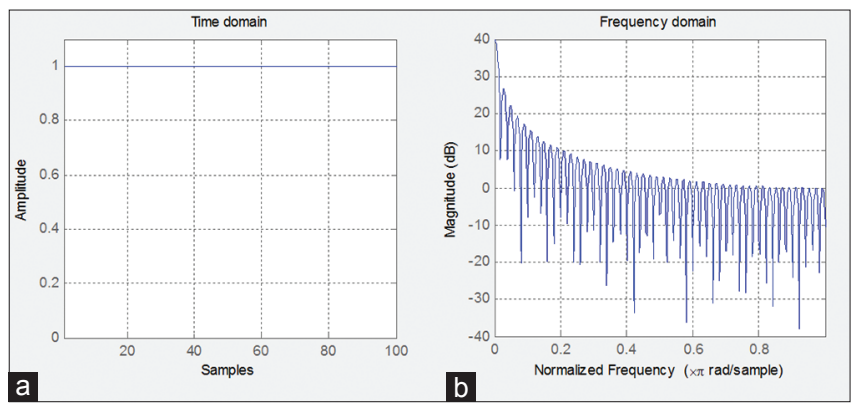

Fig. 4. (a and b) Kaiser characteristics beta $=0$

figures in frequency domain show that the relative sidelobe concentrated on $-26.8 \mathrm{~dB}$ where the latest on appears on about $-75 \mathrm{~dB}$.

Kaiser Window is an important filter window and it have weighting factor parameter. The value of beta is the parameter of Kaiser Window that affects the sidelobe attenuation in the frequency domain of the window. Fig. 4 indicates the Kaiser characteristics when beta equal to zero in which indicated that the leakage factor equal to $9.26 \%$ and the sidelobe attenuation equal to $-13.3 \mathrm{~dB}$. Fig. 5 indicates the Kaiser characteristics when beta equal to 0.5 in which indicated that the leakage factor equal to $8.48 \%$ and the

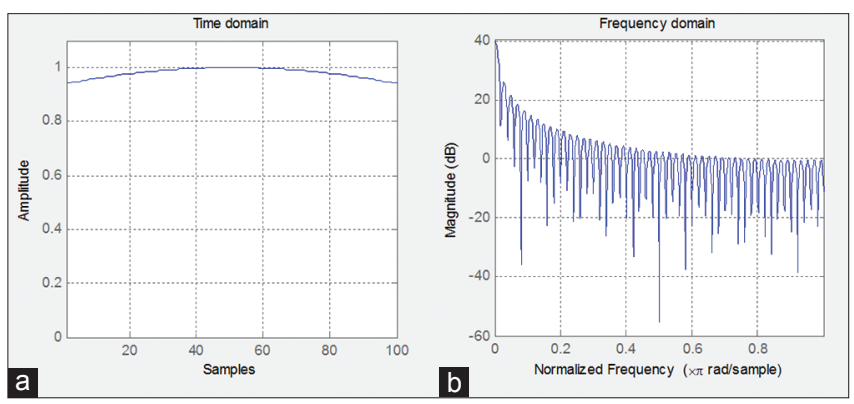

Fig. 5. (a and b) Kaiser characteristics beta $=0.5$

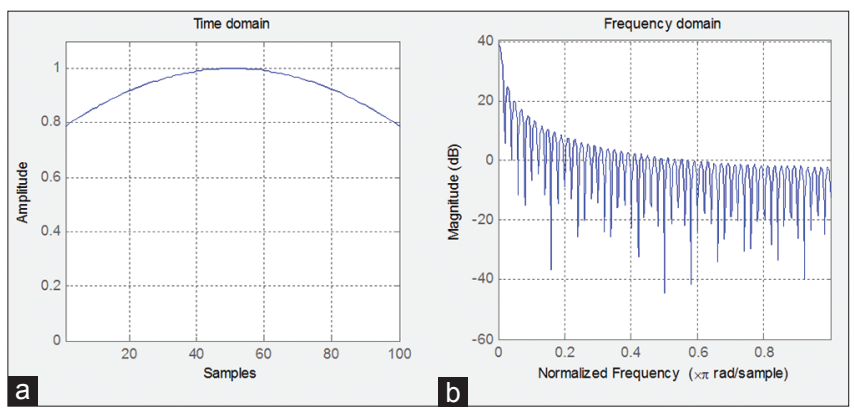

Fig. 6. (a and b) Kaiser characteristics beta $=1$

TABLE I

Kaiser Parameters of Low-beta Values

\begin{tabular}{lccc}
\hline Beta value & $\begin{array}{c}\text { Leakage } \\
\text { factor (\%) }\end{array}$ & $\begin{array}{c}\text { Sidelobe } \\
\text { attenuation dB }\end{array}$ & $\begin{array}{c}\text { Main lobe } \\
\text { width -3 dB }\end{array}$ \\
\hline 0 & 9.26 & -13.3 & 0.017578 \\
0.1 & 9.22 & -13.3 & 0.017578 \\
0.2 & 9.13 & -13.3 & 0.017578 \\
0.3 & 8.97 & -13.4 & 0.017578 \\
0.4 & 8.75 & -13.5 & 0.017578 \\
0.5 & 8.48 & -13.6 & 0.017578 \\
0.6 & 8.16 & -13.8 & 0.017578 \\
0.7 & 7.80 & -14.0 & 0.017578 \\
0.8 & 7.40 & -14.2 & 0.017578 \\
0.9 & 6.98 & -14.4 & 0.017578 \\
1.0 & 6.53 & -14.7 & 0.017578 \\
\hline
\end{tabular}

sidelobe attenuation equal to $-13.6 \mathrm{~dB}$. Table I demonstrates the Kaiser parameters of low beta values in which shows the variation of beta value from 0 to 1 with the increment of 0.1 in which the leakage factor varying from $9.26 \%$ to $6.53 \%$. In addition, the values of sidelobe attenuation is varied from -13.3 to $-14.7 \mathrm{~dB}$, with main lobe width at $-3 \mathrm{~dB}$ equal to 0.017578 for all values of beta.

Fig. 6 indicates the Kaiser characteristics when beta equal to one in which indicated that the leakage factor equal to $6.53 \%$ and the sidelobe attenuation equal to $-14.7 \mathrm{~dB}$. Fig. 7 indicates the 10 in which indicated that the leakage factor equal to $0 \%$ and the sidelobe attenuation equal to $-74.1 \mathrm{~dB}$, 
Muzhir Shaban Al-Ani: Study the Characteristics of Finite Impulse Response Filter Based on Modified Kaiser Window

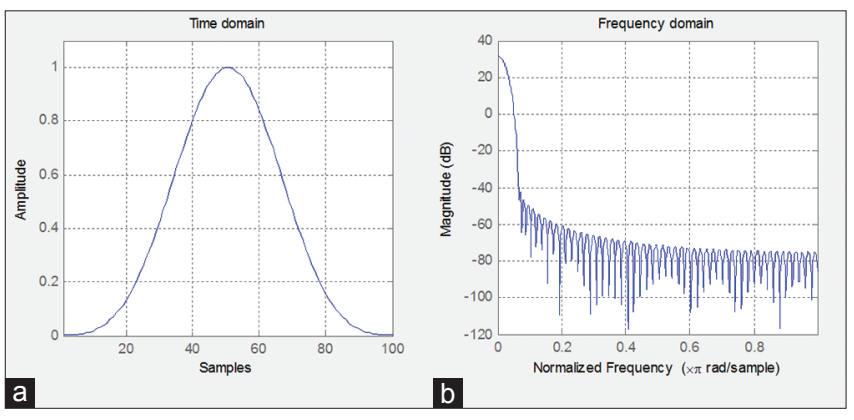

Fig. 7. $(a$ and $b)$ Kaiser characteristics beta $=10$

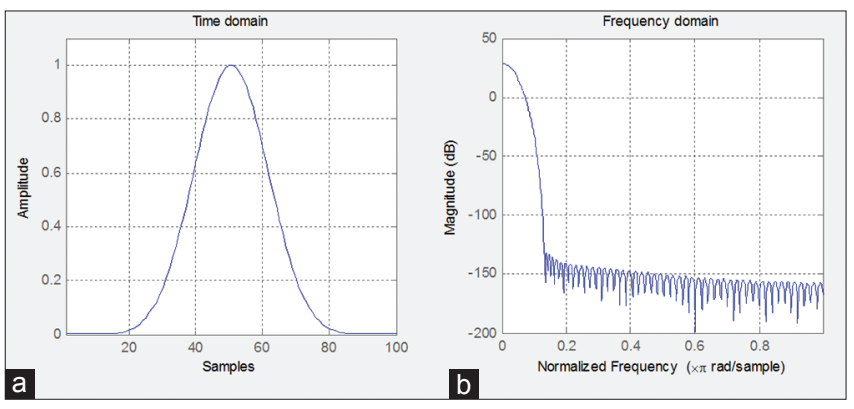

Fig. 8. ( $a$ and $b)$ Kaiser characteristics beta $=20$

in this case, the shape of the window is approach to a hat. Fig. 8 indicates the Kaiser characteristics when beta equal to 20 in which indicated that the leakage factor equal to $0 \%$ and the sidelobe attenuation equal to $-154.9 \mathrm{~dB}$, in this case, the shape of the window is stretch hat. Table II demonstrates the Kaiser parameters of high-beta values in which shows the variation of beta value from 1 to 20 with the increment of 1 , in this case, the leakage factor varying from $6.53 \%$ to $0 \%$, considering that the zero value of leakage factor starting when beta equal to 6 . In addition, the values of sidelobe attenuation is varied from -14.7 to $-154.9 \mathrm{~dB}$, with main lobe width at $-3 \mathrm{~dB}$ varying from 0.017578 to 0.048828 corresponding to the variation of the value of beta.

\section{CONCLUSION}

In this work, an adequate algorithm of Kaiser parameters is measured to be ready for the filter implementation. The framework to study the characteristics of FIR filter based on modified Kaiser Window is implemented through two scenarios. Two scenarios are implemented; First scenario used low Beta values and second scenario used high Beta values. The obtained results indicated that the implemented Kaiser Window filter has good characteristics and stable. The comparison of sidelobe attenuation and the values of beta it is possible to select an adequate and optimal

\begin{tabular}{lccc}
\multicolumn{5}{c}{ TABLE II } \\
\multicolumn{5}{c}{ Kaiser Parameters of High-beta Values } \\
\hline Beta value & $\begin{array}{c}\text { Leakage } \\
\text { factor (\%) }\end{array}$ & $\begin{array}{c}\text { Sidelobe } \\
\text { attenuation dB }\end{array}$ & $\begin{array}{c}\text { Main lobe } \\
\text { width -3 dB }\end{array}$ \\
\hline 1 & 6.53 & -14.7 & 0.017578 \\
2 & 2.47 & -18.6 & 0.019531 \\
3 & 0.61 & -24.0 & 0.021484 \\
4 & 0.12 & -30.4 & 0.023438 \\
5 & 0.02 & -37.1 & 0.025391 \\
6 & 0.00 & -44.0 & 0.027344 \\
7 & 0.00 & -51.0 & 0.029297 \\
8 & 0.00 & -58.4 & 0.031250 \\
9 & 0.00 & -66.1 & 0.033203 \\
10 & 0.00 & -74.1 & 0.035156 \\
11 & 0.00 & -82.3 & 0.035156 \\
12 & 0.00 & -90.3 & 0.037109 \\
13 & 0.00 & -98.1 & 0.039063 \\
14 & 0.00 & -105.8 & 0.041016 \\
15 & 0.00 & -113.8 & 0.041016 \\
16 & 0.00 & -122.3 & 0.042969 \\
17 & 0.00 & -130.4 & 0.044922 \\
18 & 0.00 & -138.7 & 0.044922 \\
19 & 0.00 & -147.1 & 0.046875 \\
20 & 0.00 & -154.9 & 0.048828 \\
\hline & & &
\end{tabular}

values to be used for the filter design. The obtained results indicated that an improvement of the filter characteristics during the attenuation value will increase. In addition, an improvement happens on the filter performances when filter length will increase, that is increase the number of coefficients. The implemented Kaiser Window indicated that is easy to generate many types of windows through simple modification. The sidelobe attenuation of Kaiser Window is about $-14 \mathrm{~dB}$ when beta value is 1 and this value will increase vastly up to -154.9 when beta reach to 20 .

\section{REFERENCES}

[1] M. S. M. Al-Ani and S. Lorenzo. "System implementation of LUT FFT." $6^{\text {th }}$ Mediterranean Electrotechnical Conference (MELECON'91), Yugoslavia, 22-24 May. 1991.

[2] L. Nozal, S. Lorenzo, B. Rui and M. S. M. Al-Ani. "Real time and low cost image processing architecture based on programmable logic device (PLD)." Intelligent for Mechanical System, Proceedings IROS ‘91, Osaka, Japan, pp. 279-284, 3-5 Nov. 1991.

[3] M. S. M. Al-Ani, S. Lorenzo, L. Nozal and B. Rui. "FFT LUT for image processing." The International Society for Optical Engineering (SPIE'92), Section of Algorithms Technique and Active Vision, USA, pp. 121-129, 15-20 Nov. 1992.

[4] G. I. Raho, A. J. Dawood and M. S. Al-Ani. "Real time fast algorithm of 2D DWT based DSP technology." International Journal of Application or Innovation in Engineering and Management, vol. 2, no. 10, pp. 1-5, Oct. 2013.

[5] L. Nozal, S. Lorenzo, B. Rui and M. S. M. Al-Ani. "Hardware Structure + digital signal processing on real time." International Conference on Industrial Electronics, Control, Instrumentation and 
Automation, San Diego, California, USA, pp. 1397-1402, 9-13 Nov. 1992.

[6] Q. Al-Shayea and M. S. Al-Ani. "Efficient window approach of FIR filter design (MSK2)." IJCSNS International Journal of Computer Science and Network Security, vol. 16, no. 2, pp. 63-68. Feb. 2016.

[7] Oliveira, H. Jr., H. Petraglia and A. Petraglia. "Frequency domain FIR filter design using fuzzy adapting simulated annealing." Circuits, Systems, Signal Process, vol. 28, pp. 899-911, 2009.

[8] A. A. Lanne, T. V. Merkucheva and A. I. Solonina. "Calculation of narrowband low pass filters with finite impulse response." Radioelectronic and Communication Systems, vol. 52, no. 6, pp. 311-316, 2009.

[9] A. A. Lanne and T. V. Merkucheva. "Filter with double symmetry." Radioelectronic and Communication Systems, vol. 52, no. 5, pp. 256-260, 2009.

[10] H. S. Yazdi and M. Rezaei. "The wheatstone bridge-based analog adaptive filter with application in echo cancellation." Analog Integral Circuits and Signal Processing, 16 Sep. 2009.

[11] A. Blad and O. Gustafsson. "Integer linear programmingbased bit-level optimization for high speed FIR decimation filter architectures." Circuits, Systems, Signal Process, vol. 29, pp. 81101, 2010.

[12] R. Lehto, T. Saramäki and O. Vainio. "Synthesis od wideband linear-phase FIR filters with a piecewise-polynomial-sinusoidal impulse response." Circuits, Systems, Signal Process, vol. 29, pp. 25-50, 2010.

[13] Y. C. Lim, J. W. Lee and S. W. Foo. "Quality analog scramblers using frequency response masking filter banks." Circuits, Systems, Signal Process, vol. 29, pp. 135-154, 2010.

[14] Y. Wei and Y. Lian. "Frequency response masking filters based on serial masking schemes." Circuits, Systems, Signal Process, vol. 29, pp. 7-24, 2010.

[15] Y. J. Yu and Y. C. Lim. "Optimization of linear phase FIR filters in dynamically expanding subexpression space." Circuits, Systems, Signal Process, vol. 29, pp. 65-80, 2010.

[16] L. Rosenbaum, P. Löwenborg and H. Johansson. "Two classes of cosine modulated IIR/IIR and IIR/FIR NPR filter banks." Circuits, Systems, Signal Process, vol. 29, pp. 103-133, 2010.

[17] L. Nozal, S. Lorenzo, B. Rui and M. S. M. Al-Ani. "Pipe-line programmable logic device (PLD) a new solution for image processing." SICE'91 Conference, Yonezawa, Japan, pp. 10971100, 17-19 Jul. 1991.

[18] L. Nozal, S. Lorenzo, B. Rui and M. S. M. Al-Ani. "A new vision system programable logic devices digital signal processor architecture (PLD+DSP)." International Conference on Industrial Electronics Control and Instrumentation (IECON'91), Japan, pp. 2014-2018, 28 Oct. 1 Nov. 1991.

[19] M. S. M. Al-Ani, S. Lorenzo and L. Nozal. "Fast 2D convolution filter based on LUT FFT." IEEE International Symposium on Industrial Electronics, Chain, pp. 446-449, 25-27 May. 1992.

[20] M. S. M. Al-Ani, S. Lorenzo, L. Nozal and B. Rui. "Fast image filtering implementation." Second International Conference on Document Analysis and Recognition (ICDAR'93), In: Cooperation with the IEEE Computer Society and IGS, Tsukuba Science City, Japan. 20-22 Oct. 1993.

[21] B. Rui, S. Lorenzo, L. Nozal and M. S. M. Al-Ani. "Digital signal processor accelerator board for image processing on VME bus based system." Machine Vision Application, Architectures, and System Integration, Boston, Manssachusetts, USA, pp. 85-89, 1718 Nov. 1992

[22] P. Mohindru, R. Khanna and S. S. Bhatia. "New tuning model for rectangular windowed FIR filter using fractional Fourier transform", London: SIViP, Springer-Verlag, 2013.

[23] A. Kumar, S. Sumana and G. K. Singh. "A new closed form method for design of variable bandwidth linearphase FIR filter using different polynomials." AEU-International Journal of Electronics and Communications, vol. 68, pp. 351-360, 2014.

[24] J. M. Pak, S. Y. Yoo, M. T. Lim and M. K. Song. "Weighted average extended FIR filter bank to manage the horizon size in nonlinear FIR filtering." International Journal of Control, Automation, and Systems, vol. 13, no. 1, pp. 1-8, 2015.

[25] P. L. Barnes and G. C. Verghese. "Uniform FIR approximation of causal wiener filters, with applications to causal coherence." Signal Processing, vol. 122, pp. 129-137, 2016.

[26] X. Huang, Y. Wang, Z. Yan, H. Xian and M. Liu. "Closed-form FIR filter design with accurately controllable cut-off frequency, circuits syst signal process," New York: Springer Science and Business Media, 2016. Available: https://link.springer.com/article/10.1007/ s00034-016-0330-7. [Apr. 27, 2016].

[27] J. M. Pak, P. S. Kim, S. H. You, S. S. Lee and M. K. Song. "Extended least square unbiased FIR filter for target tracking using the constant velocity motion model. International Journal of Control, Automation and Systems, vol. 15, no. X, pp. 1-5, 2017.

[28] A. Boukharouba. "Smoothed rectangular function-based FIR filter design, circuits system signal process," New York: Springer Science and Business Media, 2017. Available: https://link.springer. com/article/10.1007/s00034-017-0529-2. [Feb. 21, 2017]. 